\title{
Radiolários: estado do conhecimento e aplicações às geociências
}

\author{
Karlos Guilherme Diemer Kochhann \\ Lab. Micropaleontologia, Univ. do Vale do \\ Rio dos Sinos (Unisinos). São Leopoldo, RS. \\ k.kochhann@gmail.com
}

\begin{abstract}
RADIOLARIANS: STATE OF KNOWLEDGE AND THEIR APPLICATIONS TO GEOSCIENCES. Radiolarians are planktonic protists with wide spatial and temporal distribution (throughout oceanic basins as well as through geological time). The protoplasmic body is divided in endoplasm and ectoplasm, the former related to reproductive functions and the latter to the regulation of the position in the water column, digestion and breathing. The endoplasm and the ectoplasm are separated by the capsular membrane, which is an exclusive characteristic of the group. Their skeletons are composed by amorphous silica, that enables their preservation in marine sediments deposited below de calcite compensation depth (CCD). These characteristics allow to apply fossil radiolarians to solve several problems in geosciences, as in paleobiogeography, paleogeographic reconstruction of tectonically complex areas, biostratigraphy and paleoceanography/paleoclimatology. Citation: Kochhann K.G.D. 2011. Radiolários: estado do conhecimento e aplicações às geociências. Terræ Didatica, 7(1):30-40< http://www.ige.unicamp.br/terraedidatica/>
\end{abstract}

KEYWORDS radiolarians, geosciences, paleobiogeography, bioestratigraphy, paleoceanography.

\begin{abstract}
RESUMO Radiolários são protistas planctônicos com ampla distribuição espacial e temporal (entre bacias oceânicas e através do tempo geológico). O corpo protoplasmático pode ser dividido em endoplasma e ectoplasma, sendo que o primeiro desempenha principalmente funções reprodutivas e o segundo desempenha funções na manutenção da posição do organismo na coluna da água, digestão e respiração. Endoplasma e ectoplasma são separados pela membrana capsular, característica que diferencia os radiolários dos demais protistas. Seus esqueletos são compostos por sílica amorfa, o que permite sua preservação em sedimentos marinhos depositados abaixo da zona de compensação da calcita (CCD - Calcite Compensation Depth). Estas características permitem a aplicação do grupo na resolução de diversos problemas geológicos, como, por exemplo, na paleobiogeografia, reconstituições paleogeográficas de áreas tectonicamente complexas, bioestratigrafia e paleoceanografia/paleoclimatologia.
\end{abstract}

PALAVRAS-CHAVE radiolários, geociências, paleobiogeografia, bioestratigrafia, paleoceanografia.

\section{Introdução}

Radiolários são protistas planctônicos amplamente distribuídos pelos oceanos modernos e do passado geológico. Esta vasta abrangência temporal e espacial permite que estes organismos sejam utilizados na resolução de diversos problemas geológicos. Neste escopo se insere o presente estudo, que apresenta uma síntese do estado de conhecimento sobre o grupo, historicamente marcado pela carência nos seus aspectos biológicos, e discute algumas de suas aplicações às geociências.

Os radiolários, além da ampla distribuição espacial e temporal, possuem larga distribuição através da coluna da água, habitando desde águas próximas à superfície até águas profundas. Seus esqueletos são formados por sílica amorfa e podem viver como formas solitárias (com tamanho variando de $30 \mu \mathrm{m}$ a $2 \mathrm{~mm}$ ) e/ou em colônias macroscópicas interconectadas. O registro fóssil dos radiolários se estende do Cambriano (540 Ma) ao Recente (De Wever et al. 2001).

A célula possui uma massa central de citoplasma (endoplasma), contendo um ou mais núcleos, mitocôndrias, dictiossomos e o axoplasto, o qual origina os axópodes (De Wever et al. 2001). A camada periférica de citoplasma (ectoplasma), contém alvéolos em formato de bolhas, axópodes radiais, 
rizópodes em redes, vacúolos (veja Boltovskoy \& Pujana 2007), e é separada do endoplasma por uma parede capsular central perfurada (membrana capsular) (De Wever et al. 2001) (Fig. 1). A presença da membrana capsular é uma das características que diferencia os radiolários dos demais protistas (Eilert et al. 2004). apresentar simbiose com algas. Seu registro fóssil estende-se do Cambriano ao Recente. Neste artigo são discutidos, principalmente, radiolários Polycystina.

A superordem Phaeodaria caracteriza-se pela cápsula central perfurada por apenas três poros e ausência de simbioses. Seu registro fóssil possui

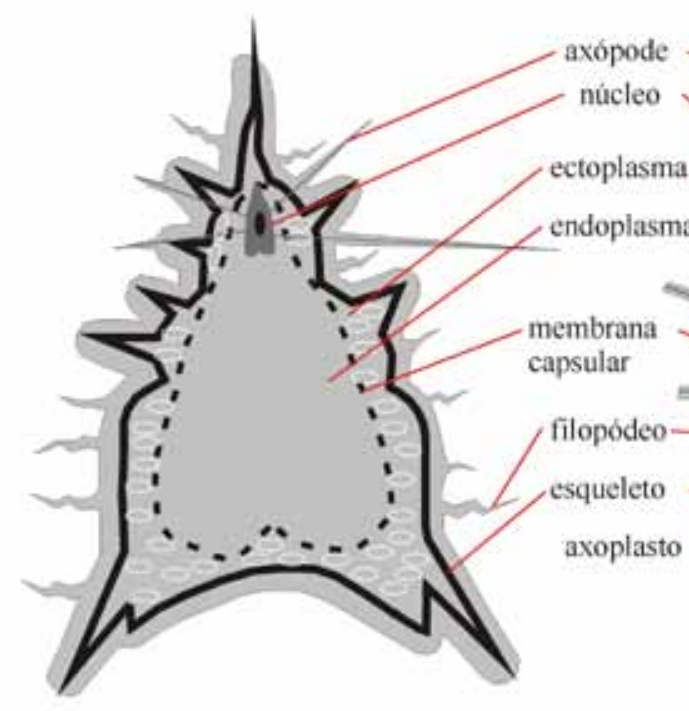

NASSELLARIA

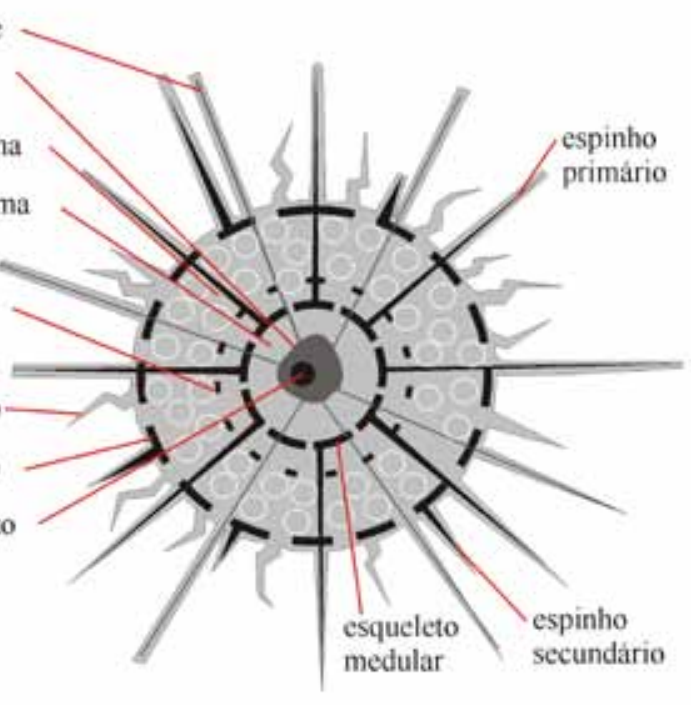

SPUMELLARIA

Figura 1. Organização básica da célula de radiolários (Modif. de Boltovskoy \& Pujana 2007)

Taxonomicamente, os radiolários são agrupados na classe Actinopoda e subclasse Radiolaria (Tab. 1). Esta compreende organismos que apresentam axópodes e, em muitos grupos, membrana capsular. A subclasse divide-se em quatro superordens: Polycystina, Phaeodaria, Heliozoa e Acantharia (De Wever et al. 2001).

A superordem Polycystina é composta por espécies que possuem a cápsula central total ou parcialmente perfurada (p. ex. Spumellaria), podendo

Tabela 1. Classificação Zoológica (Modif. de De Wever et al. 2001)

\begin{tabular}{l|c}
\hline Reino: & Protista \\
\hline Sub-reino: & Protozoa \\
\hline Filo: & Sarcomastigophora \\
\hline Subfilo: & Sarcodina \\
\hline Classe: & Actinopoda \\
Subclasse: & Radiolaria \\
Superordens: & Polycystina \\
& Phaeodaria \\
& Heliozoa \\
& Acantharia \\
\hline
\end{tabular}

raras ocorrências no Maastrichtiano (Dumitrica \& Hollis 2004) e Paleógeno, tornando-se mais abundante no Neógeno e Quaternário.

Os representantes da superordem Heliozoa apresentam o citoplasma dividido em endo- e ectoplasma e esqueleto frágil ou ausente, fato que contribui para a raridade do seu registro fóssil.

Nas espécies da superordem Acantharia a cápsula central é perfurada por numerosos poros pequenos e ocorrem hábitos simbióticos com algas. Seus esqueletos são compostos por celestina (a composição química do esqueleto é uma característica importante em níveis taxonômicos altos - Campbell 1954), não apresentando registro fóssil.

A superordem Polycystina congrega as seguintes ordens: Archaeospicularia (Fig. 2a) (Meso-Cambriano? ao Siluriano?); Albaillellaria (Fig. 2c) (Ordoviciano?-Siluriano ao final do Permiano ou Eo-/Mesotriássico); Latentifistularia (Fig. 2b) (Neocarbonífero? ao final do Permiano); Spumellaria (Fig. 2d) (Paleozoico ao Recente); Collodaria (Fig. 2e) (Eoceno? ao Recente); Entactinaria (Fig. 2f) (Paleozoico ao Recente); Nassellaria (Fig. 
2g) (Devoniano ao Recente, mas de acordo com alguns autores, Eotriássico ao Recente). Algumas características inerentes a estas ordens serão abordadas no decorrer do texto, principalmente referentes às ordens Spumellaria e Nassellaria.

\section{Breve histórico}

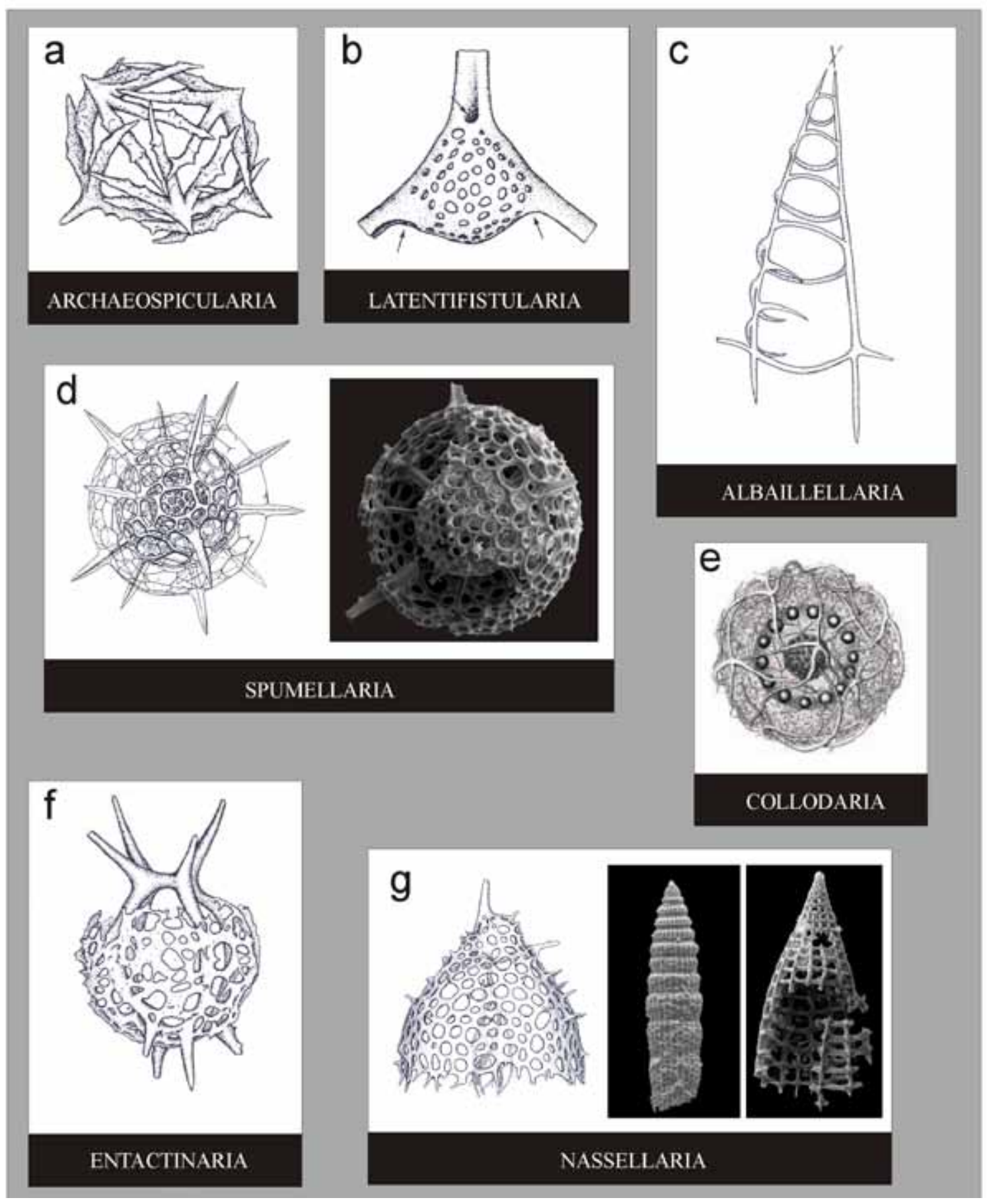

Figura 2. Ordens de radiolários Polycystina: a-Archaeospicularia; $b$-Latentifistularia; $c$-Albaillellaria; $d$-Spumellaria; e-Collodaria; $f$-Entactinaria; g-Nassellaria. Imagens modificadas de De Wever et al. (2001), Boltovskoy \& Pujana (2007) e Aumond et al. (2009) 
um esquema de classificação que, embora atualmente seja considerado artificial, tem algumas de suas propostas ainda utilizadas.

Em meados do século XX o interesse pelo grupo voltou a aumentar, principalmente devido a estudos de cunho estratigráfico. Boltovskoy \& Pujana (2007) estimam que, do total de trabalhos publicados sobre radiolários, mais de $50 \%$ referem-se a faunas do Cenozoico, de 30-35\% a faunas do Mesozoico e aproximadamente $10 \%$ a faunas do Paleozoico. No que concerne aos aspectos biológicos do grupo, o número de trabalhos publicados vem diminuindo drasticamente nas últimas décadas, necessitando-se de estudos mais aprofundados em alguns tópicos.

\section{0 organismo em vida}

\section{Estrutura celular}

Radiolários em vida não têm sido tão extensivamente estudados quanto seus correspondentes no registro fóssil. Muitos dos estudos envolvendo radiolários atuais apresentam caráter biogeográfico ou abordam o padrão de distribuição vertical das espécies nas massas de água (Boltovskoy 1998, Boltovskoy et al. 1993, Boltovskoy \& Riedel 1987). Recentemente, estudos também vêm sendo desenvolvidos sobre a taxa e o modo de crescimento do esqueleto, biologia e morfologia (e.g. Ogane et al. 2009, Jacquot Des Combes \& Abelmann 2009).

$\mathrm{O}$ endoplasma geralmente é mais denso que o ectoplasma (Campbell 1954). O ectoplasma desempenha importantes funções na manutenção da posição do organismo na coluna da água, digestão, respiração, entre outras. Já o endoplasma desempenha principalmente funções reprodutivas (Campbell 1954).

A cápsula central é composta pelo endoplasma, possuindo inclusões, um ou mais núcleos e o axoplasto. Caso um indivíduo seja reduzido apenas à cápsula central, esta estará apta a regenerar todas as demais partes do organismo (De Wever et al. 2001).

A membrana capsular (Fig. 3) limita externamente a cápsula central e é pigmentada, sendo composta por quitina ou pseudoquitina, fusules (pequenos tubos pelos quais passam os filamentos axopodiais) e fissuras capsulares. Os espumelários apresentam numerosos poros distribuídos por toda a membrana, enquanto os nasselários apresentam poros concentrados em uma porção da membrana capsular, possuindo fusules grandes, distribuídos em um cone chamado podocone (De Wever et al. 2001).

O endoplasma apresenta um ou mais núcleos, praticamente centrais. A organização nuclear é controlada pela morfologia axopodial e pela morfologia do esqueleto. $\mathrm{O}$ endoplasma também contém as mitocôndrias, o dictiossomo, os vacúolos e o axoplasto, do qual se originam os axópodes (sendo os axópodes principais denominados axoflagelos). Em indivíduos pertencentes à ordem Nassellaria, o axoplasto é sempre externo ao núcleo (De Wever et al. 2001).

Os axópodes são prolongamentos citoplasmáticos longos, finos e rígidos. São retráteis em condições de stress e funcionam como mecanismo para

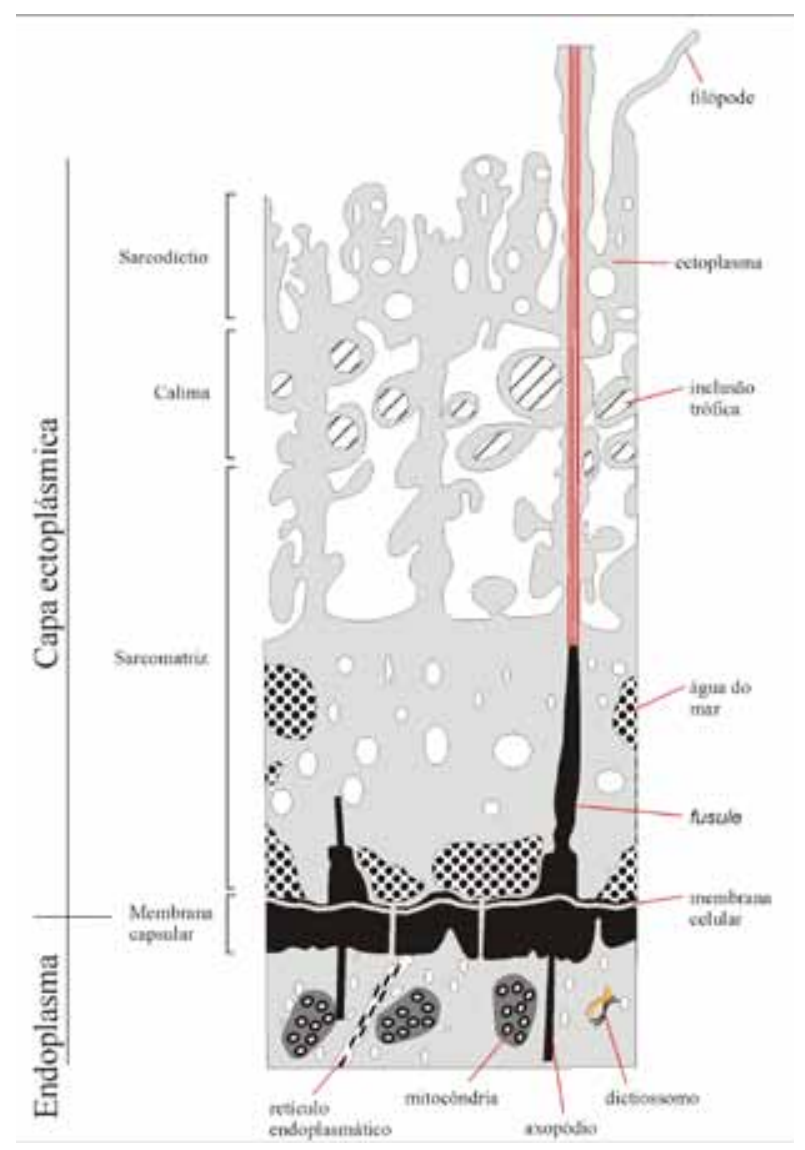

Figura 3. Relação entre o endoplasma, membrana capsular, membrana celular, fusules e a capa ectoplásmica (Modificado de De Wever et al. 2001) 
a captura de presas (De Wever et al. 2001) (Fig. 4). Além de serem úteis na captura de nutrientes, estes podem auxiliar na manutenção da posição vertical na coluna da água, bem como, no transporte de sílica obtida por meio de presas, e.g. diatomáceas (Cachon \& Cachon 1971).

O corpo extra-capsular é composto pelo ectoplasma com suas diversas inclusões, bem como, pelos pseudópodes. Divide-se em sarcomatriz, calima e sarcodíctio (ordem: do mais interno ao mais externo) (De Wever et al. 2001). Pseudópodes são projeções citoplasmáticas externas à célula (filópodes, axópodes e axoflagelos). Filópodes são finos prolongamentos de citoplasma de base anastomosada que são utilizados para engolfar presas, levando-as até os vacúolos digestivos, e possuem origem no sarcodíctio.

O esqueleto dos radiolários é composto por sílica amorfa $\left(\mathrm{SiO}_{2}\right.$, n. $\left.\mathrm{H}_{2} \mathrm{O}\right)$ autigênica, retirada diretamente da água do mar. Durante a vida do organismo o esqueleto é envolto por material citoplasmático, com algumas partes sendo, por vezes, inseridas no endoplasma e no núcleo. Quando presentes, os espinhos são envoltos pelos pseudópodes.

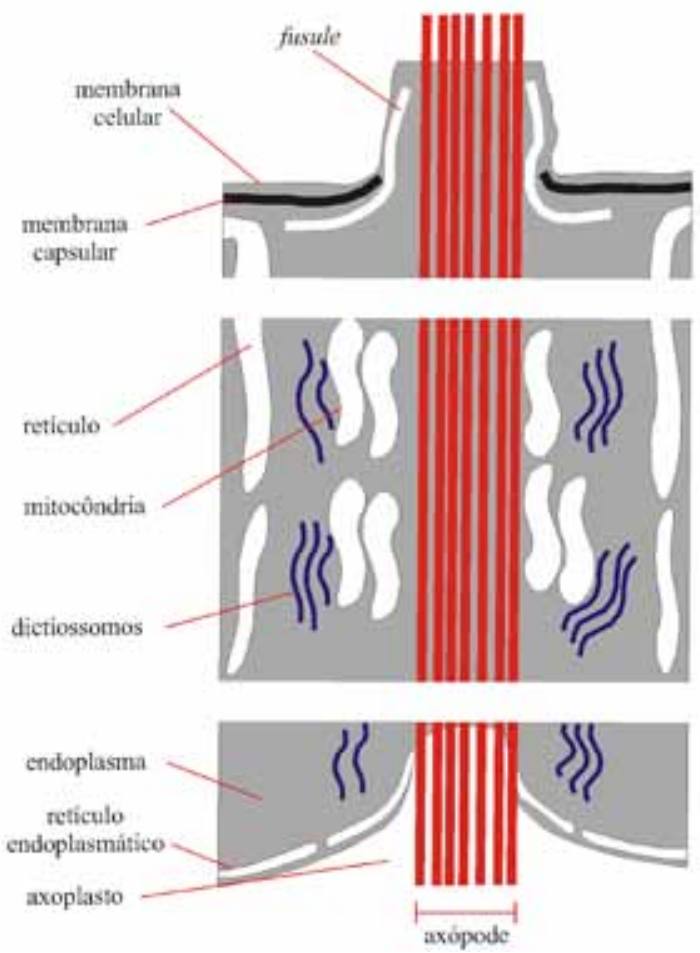

Figura 4. Seção esquemática de um axópode na cápsula central de radiolários Polycystina (Extr. De Wever et al. 2001)
Devido a este fato, o esqueleto dos radiolários não está sujeito à dissolução em águas subsaturadas em sílica durante o período de vida do organismo. O crescimento do esqueleto dos radiolários é descontínuo, sendo caracterizado por fases de formação rápida do esqueleto, alternadas com períodos de baixa taxa de crescimento (De Wever et al. 2001). Segundo Campbell (1954), a secreção de sílica e outras substâncias minerais ocorre principalmente no ectoplasma.

\section{Aspectos biológicos}

O crescimento do esqueleto dos radiolários segue um padrão geométrico característico para cada espécie. Existem duas linhas de hipóteses propostas para a compreensão do desenvolvimento do esqueleto: (i) baseada puramente em leis físicas (Thompson 1942) e, (ii) baseada em interações biológicas (Anderson 1986).

A proposta de desenvolvimento esqueletal baseada em princípios físicos, proposta por Thompson (1942), supõe, entre outras hipóteses, que a película citoplásmica poderia ser composta por vesículas justapostas em um padrão prismático, alcançado de acordo com as tensões superficiais, favorecendo a precipitação de sílica nos pontos de junção. Este padrão poderia gerar padrões de poros hexagonais e paredes esponjosas nos esqueletos de radiolários.

No que concerne a hipótese de interações biológicas, os estágios de desenvolvimento esqueletal são: juvenil, intermediário e adulto, podendo crescer: i) por meio do desenvolvimento de bridges sobre a concha porosa e ii) pelo espessamento de estruturas secundárias. Há uma íntima relação entre a arquitetura celular e a organização do esqueleto, fato que favorece a segunda hipótese em detrimento da primeira. Os espumelários apresentam crescimento centrífugo, sendo a concha interna a primeira a ser formada. Os nasselários começam a desenvolver o esqueleto em torno de uma espícula central localizada no céfalo (ordem dos segmentos: céfalo, tórax, abdômen e segmentos pós-abdominais). O crescimento do esqueleto dos radiolários é descontínuo, controlado pela fisiologia da célula e por uma janela de temperatura estreita, porém, tolera fortes variações de salinidade (De Wever et al. 2001).

$\mathrm{O}$ formato dos esqueletos e as estruturas geradas são bastante diversificados, sendo de extrema 
importância para a classificação a nível genérico por retratarem a grande variedade de formas do grupo (Campbell 1954). A parede das conchas pode ser treliçada, perfurada ou esponjosa. Os espinhos podem ser circulares, sulcados, trifacetados, ou tetrafacetados em seção cruzada. As ordens Spumellaria, Entactinaria, Archaeospicularia e Collodaria apresentam esqueletos com simetria radial, enquanto, as ordens Nassellaria, Albaillellaria e Latentifistularia possuem esqueletos com simetria não-esférica, usualmente bilateral. Estimativas do tempo de vida dos radiolários, observadas em laboratório, giram em torno de três semanas (De Wever et al. 2001).

Muitos pesquisadores assumem que os radiolários se reproduzem de forma sexuada, porém, diversas espécies já estudadas reproduzem-se assexuadamente. A reprodução assexuada pode ocorrer por fissão binária ou fissões múltiplas. A fissão binária ocorre quando a célula inicial se divide em duas, porém apenas uma herda a estrutura esqueletal e a outra célula precisa reconstruí-lo. A fissão múltipla, por sua vez, ocorre por esporogênese e pode também representar o estágio inicial da reprodução sexuada. Após sua liberação na água, devido à ruptura da cápsula central, os zoósporos podem tanto formar outra célula, quanto se combinar para a formação de um zigoto (De Wever et al. 2001).

Em adição à nutrição derivada das algas endosimbiônticas, a maioria dos radiolários consomem uma ampla variedade de presas algálicas e animais. Algumas espécies são onívoras e predam silicoflagelados, tintinídeos, diatomáceas, algas, bactérias, copépodos e pequenos cnidários. Alguns grupos possuem preferências alimentares, e.g. espumelários espinhosos parecem apresentar preferência pela predação de zooplâncton, enquanto espumelários não-espinhosos tendem a preferir algas. A digestão ocorre nos vacúolos digestivos (De Wever et al. 2001). Armstrong e Brasier (2005) afirmam que há uma tendência de aumento no tamanho dos indivíduos, de herbívoros para onívoros.

Quanto à simbiose, entendem-se por simbiontes, algas que estejam contidas nos vacúolos citoplasmáticos. Nos Polycystina, os dinoflagelados são os simbiontes mais comuns, podendo ocorrer também prymnesiófitas. O hospedeiro e os simbiontes morrem ao mesmo tempo. A transferência de $\mathrm{C}^{14}$ por parte dos simbiontes é de grande importância para a nutrição dos radiolários, permitindo que sobrevivam por longos períodos sem nutrição externa. A simbiose entre radiolários e algas pode ser confirmada pela correlação positiva entre as curvas de abundâncias de radiolários e fitoplâncton ao longo do tempo geológico (De Wever et al. 2001).

Por décadas, pensava-se que os radiolários eram flutuadores passivos, sem a mínima capacidade de movimentação, entretanto, estudos de laboratório demonstraram a capacidade de rastejar bem como, fixar-se a objetos flutuantes. A flutuabilidade dos radiolários é possível graças ao conteúdo lipídico dos vacúolos. Para mergulhar, os radiolários ejetam o conteúdo vacuolar, tornando assim o citoplasma mais denso. Os axopódios desempenham um importante papel na manutenção da posição do organismo na coluna da água (expansão e contração dos vacúolos). Caso haja perda de ectoplasma e axopódios, o organismo tende a afundar. A movimentação vertical dos radiolários na coluna da água também pode se dar por mudanças na temperatura e/ou movimentos de massas de água (De Wever et al. 2001).

\section{Ecologia e paleoecologia}

Embora a sobrevivência de radiolários seja amplamente favorecida em águas marinhas de salinidade média, estes não estão ausentes em áreas de baixas (não muito inferiores a $30 \%$-Boltovskoy \& Pujana 2007) e altas salinidades (Campbell 1954). Poucas formas apresentam preferências por águas costeiras ou neríticas e, alguns indivíduos podem ser carregados acidentalmente para grandes baías (Campbell 1954). Boltovskoy et al. (2003) reportaram a primeira, e única, taxocenose monoespecífica de Lophophaena rioplatensis em águas salobras do estuário Rio del Plata e águas costeiras próximo ao Mar del Plata, suportando salinidades de até $15,4 \%$. As áreas de maior abundância de radiolários, geralmente estão associadas a zonas oceânicas de ressurgência - upwelling zones - ricas em nutrientes (Boltovskoy \& Pujana 2007).

A distribuição geográfica dos radiolários apresenta, em geral, as mesmas relações que outros organismos planctônicos, evidenciando principalmente padrões de correntes oceânicas (Campbell 1954). Como diversos organismos marinhos, os radiolários atingem sua máxima diversidade em águas tropicais, enquanto as massas de águas frias, de latitudes mais elevadas, são caracterizadas por 
grande abundância e baixa diversidade de formas (Campbell 1954).

Gradientes de temperatura, sílica e outros macronutrientes provavelmente influenciam as variações latitudinais na abundância de radiolários vivos (Abelmann \& Gowing 1996). Assim como com foraminíferos, algumas espécies de águas frias que vivem em águas superficiais, de províncias subpolares, podem aparecer a grandes profundidades próximas ao equador (Boltovskoy \& Pujana 2007).

Bogdanov \& Vishnevskaya (1992) observaram uma mudança no habitat preferencial dos radiolários, de plataformas carbonáticas rasas no Paleozoico para o exclusivo domínio oceânico no Recente.

Em estudos paleoecológicos, são utilizadas, amplamente, funções de transferência, originalmente propostas por Imbrie \& Kipp (1971). O princípio desta técnica está na relação entre medidas diretas de condições ambientais (e.g. temperatura) e as porcentagens de espécies atuais relacionadas a determinados intervalos destas condições. Estes dados são extrapolados para o registro geológico, baseando-se em similaridades ecológicas entre as espécies atuais e fósseis. Este fato ocasiona uma perda de confiabilidade quando se aplicam funções de transferência a sucessões sedimentares mais antigas (Boltovskoy \& Pujana 2007).

\section{Da água aos sedimentos e às rochas}

Aproximadamente $10 \%$ do volume de sílica de origem biogênica acumulada em sedimentos marinhos de fundo são representados por radiolários, sendo o restante, composto por diatomáceas e espículas de esponjas. Normalmente, apenas um pequeno número das espécies observadas no plâncton não apresenta registro nos sedimentos, o que demonstra que o registro sedimentar reflete a biocenose planctônica e, portanto, constitui um importante registro paleoceanográfico (De Wever et al. 2001). Os radiolários distribuem-se através da coluna da água desde a superfície até aproximadamente $5000 \mathrm{~m}$ de profundidade, ocorrendo em maior abundância entre 50 e $100 \mathrm{~m}$ de profundidade (Fig. 5). Até mesmo variações morfológicas podem ser observadas entre espécies que habitam águas superficiais quentes (esqueletos delicados) e espécies que habitam águas profundas e frias (esqueletos mais robustos) (Campbell 1954).
Estudos têm mostrado que variações temporais em faunas de radiolários podem ser utilizadas como indicadores de paleoprodutividade. Um grande número de espécies parece estar relacionado à alta produtividade, podendo, o fluxo vertical de radiolários através da coluna da água aos sedimentos, refletir os padrões de produtividade primária. Historicamente, a abundância de radiolários foi associada a eventos vulcânicos, que seriam responsáveis por saturar a água do mar em sílica, porém, esta relação é considerada, hoje, incorreta, sendo a abundância de radiolários aparentemente relacionada com a alta disponibilidade de nutrientes. É possível, entretanto, que um aumento no vulcanismo torne a água menos

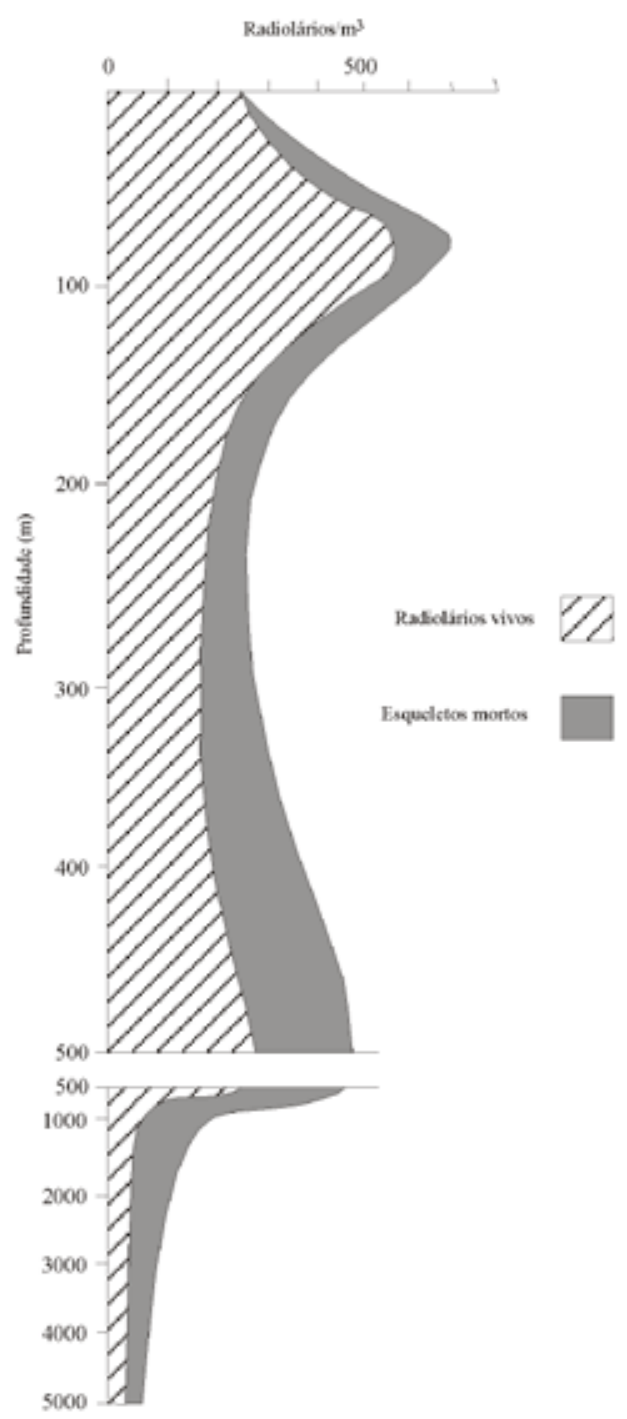

Figura 5. Distribuição vertical de radiolários vivos e mortos através da coluna da água em uma estação no Oceano Pacífico Central (Modif. Funnell \& Riedel 1971) 
depletada em sílica, intensificando a preservação da sílica amorfa. (De Wever et al. 2001). Radiolários estão, muitas vezes, associados a depósitos ricos em matéria orgânica, sendo que ambos necessitam de alta produtividade biótica superficial para sua formação (Sandoval et al. 2008).

A dissolução dos esqueletos silicosos atua como um importante fator limitante na sedimentação dos radiolários. Os padrões de preservação independem da profundidade da lâmina d'água e a dissolução dos esqueletos continua a agir abaixo da interface água/ sedimento, quando estes estão expostos à água subsaturada em sílica (De Wever et al. 2001). Por vezes, os esqueletos de radiolários que apresentam melhor preservação são aqueles que afundam rapidamente em direção ao fundo oceânico, inseridos em pelotas fecais de copépodes (Swain 1977)

A transformação das assembleias de radiolários depositadas nos sedimentos em rocha se processa por meio da dissolução e da recristalização dos esqueletos. Opala-A (opala amorfa) é transformada em opala-CT (opala + tridimita + cristobalita) e, finalmente, calcedônia e/ou quartzo (variando de acordo com o grau de liberdade). A transformação de opala em quartzo é realizada através de um estágio líquido (Mizutani 1966), sendo governada principalmente pela temperatura e, em menor grau, pelo tempo e pela composição do sedimento circundante. A epigenia auxilia na preservação dos esqueletos, através da substituição da sílica por calcita, pirita, esmectita, zeolita, e, eventualmente, rodocrosita, kutnahorita e clinoptilolita (De Wever et al. 2001).

Radiolaritos são rochas compostas por camadas de chert vermelho ou verde alternadas com finas camadas de pelitos. As camadas de chert são formadas pela vasa silicosa (no caso de radiolaritos, principalmente por radiolários) que são submetidas a processos diagenéticos. Bacias tetianas que apresentam radiolaritos mesozóicos são geralmente alongadas, rasas, relativamente pequenas e localmente restritas, com a deposição dessas rochas ocorrendo periodicamente (De Wever et al. 2001).

A abundância de radiolários pode variar consideravelmente, de acordo com a composição e origem dos depósitos sedimentares nos quais as assembléias estão contidas.

\section{Radiolários e o Tempo Geológico}

Os registros mais antigos de radiolários remetem ao Cambriano da Austrália, apresentando formas cônicas, esferas fechadas e espículas isoladas (Won \& Below 1999). Alguns autores sugerem que existe uma transição no que concerne o modo de vida do grupo, de bentônico para planctônico (Armstrong \& Brasier 2005). No Paleozoico e Mesozoico surgiram diversos grupos de Spumellaria e Albaillellaria (Swain 1977).

Uma dramática redução no número de espécies de águas frias ocorreu durante o Permiano e o Triássico, provavelmente, devido à clausura tectônica de muitas bacias paleozóicas, à reorganização e redução no número de correntes oceânicas superficiais e à eutrofização devido à glaciação permiana (Hallam \& Wignall 1997). Os primeiros registros de Nassellaria remetem ao Triássico e praticamente a metade dos grupos de radiolários modernos surgiu no Mesozoico (Armstrong \& Brasier 2005).

O registro fóssil sugere que, diferentemente das diatomáceas e silicoflagelados, os radiolários não se irradiaram com o esfriamento global durante a Era Cenozoica (Armstrong \& Brasier 2005). Uma provável explicação para este padrão seria a redução, em área, do cinturão equatorial de águas quentes (onde os radiolários atingem sua máxima diversidade) durante este intervalo de tempo (Armstrong \& Brasier 2005). Durante o Cenozoico, os radiolários também mostram uma progressiva diminuição na silicificação de seus esqueletos (Casey et al. 1983), provavelmente relacionada à maior competição por sílica nos oceanos entre radiolários e diatomáceas (Racki 1999).

\section{Algumas aplicações às Geociências}

As preferências paleo-/ecológicas dos radiolários, bem como as abrangências estratigráficas dos taxa, permitem sua aplicação em diversas áreas das geociências.

Fundamentados na existência de provincionalismo biogeográfico, Pessagno \& Blome (1986) propuseram um modelo paleobiogeográfico para o Jurássico Superior a Médio, utilizando as ocorrências de Ristola/Pantanelliidae e Parvicingula (Fig. 6). De acordo com estes autores, o Reino Tetiano (latitudes baixas) seria caracterizado por um aumento na abundância e na diversidade de Pantanelliidae e/ou Ristola, enquanto o Reino Boreal (latitudes médias a altas) seria caracterizado pela dominância de Parvicingula. Analogamente, no Quaternário, Nigrini (1967) reporta um forte controle latitudinal na distribuição de diversas espécies no Oceano 
Índico. Estudos desta temática podem contribuir para o melhor conhecimento de terrenos antigos, principalmente de evolução tectônica complexa (e.g. Carter \& Haggart 2006), ou, ainda, para a inferência de períodos influenciados, dominantemente, por correntes oceânicas de altas ou baixas latitudes em determinada bacia sedimentar (e.g. Kochhann de compensação da calcita (CCD - Calcite Compensation Depth), onde os microfósseis carbonáticos são destruídos. De Wever et al. (2001) apresentam uma compilação de diversos esquemas bioestratigráficos (paleozoicos, mesozoicos e cenozoicos) formulados com base em radiolários.

Um dos tópicos mais controversos com relação

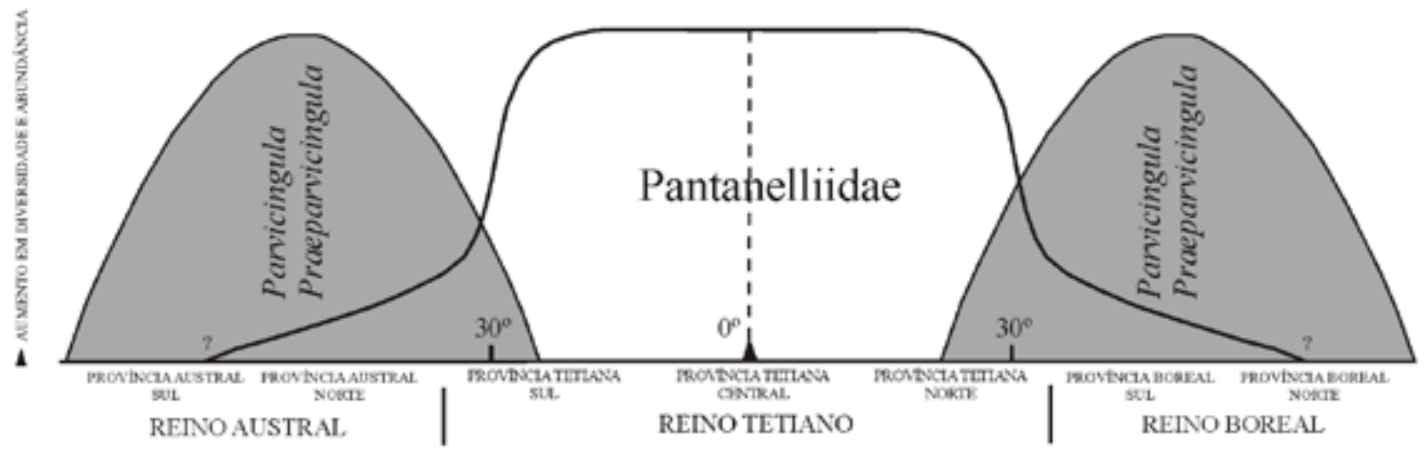

Figura 6. Modelo paleobiogeográfico proposto por Pessagno \& Blome (1986) e modificado por Pessagno \& Hull (2002)

et al. 2009).

As preferências paleoecológicas dos radiolários podem indicar diversos aspectos paleoceanográficos e/ou paleoclimáticos. A abundância e a composição de assembleias podem ser usadas para reconstruir mudanças na paleoprodutividade primária nas águas superficiais (e.g. TSRI (termocline/surface radiolarian index) $=$ taxa que vivem abaixo da termoclina/ taxa que habitam águas superficiais (Jacquot Des Combes et al. 1999)). Assembleias de radiolários também permitem estimar a paleotemperatura das massas de água oceânicas superficiais, por meio de análises fatoriais (e.g. Cortese \& Abelmann 2002). No Brasil, vários estudos foram realizados com base em radiolários cenozoicos, principalmente quaternários, na bacia de Pelotas, abordando as preferências termais das assembleias (Kotzian 1984, Eilert 1985, Kotzian \& Eilert 1985, Kotzian et al. 1987).

Devido ao longo registro geológico apresentado pelos radiolários, que abrange todo o Fanerozoico, o grupo apresenta ampla utilização em estudos bioestratigráficos. Embora os radiolários apresentem uma ampla distribuição geográfica, a distribuição de muitos taxa é controlada por faixas latitudinais, tornando necessária a aplicação de esquemas bioestratigráficos distintos para áreas localizadas em diferentes latitudes (e.g. Takemura 1992, Funakawa \& Nishi 2005). Além disso, a composição silicosa dos esqueletos permite a sua aplicação bioestratigráfica em sedimentos depositados abaixo da zona à ocorrência de radiolários no Brasil provém da Bacia Sanfranciscana (Kattah \& Koutsoukos 1992). Segundo Eilert (2001), a presença de radiolários em uma fina camada de chert $(\sim 3 \mathrm{~cm})$ sugere, fortemente, a influência marinha em uma sucessão sedimentar historicamente considerada continental. Entretanto, pelo fato dos espécimes estarem mal preservados, um estudo mais detalhado desta fauna é necessário para a resolução dos conflitos ainda existentes.

\section{Agradecimentos}

O autor manifesta seus agradecimentos aos doutores Simone Baecker-Fauth, Cristianini Trescastro Bergue e Gerson Fauth pela leitura crítica do manuscrito e pelo incentivo à publicação deste trabalho. A revisão crítica do Dr. Antonio Carlos Sequeira Fernandes contribuiu de forma significativa para o aprimoramento da versão final deste manuscrito.

\section{Referências}

Abelmann A., Gowing M.M. 1996. Horizontal and vertical distribution pattern of living radiolarians along a transect from the Southern Ocean to the South Atlantic Subtropical Region. Deep Sea Research - Part I, 43:361-382.

Anderson O.R. 1986. Silicification in Radiolaria - deposition and ontogenetic origin of forms. 
In: Leadbeater B.S.C., Riding R. eds. 1986. Biomineralization in lower plants and animals. Oxford: Clarendom Press. p. 375-391. (The Systematics Association, Special Volume).

Armstrong H., Brasier M. 2005. Microfossils. Oxford: Blackwell Publishing. 296 pp.

Aumond G.N., Kochhann K.G.D., Florisbal L.S., Baecker-Fauth S., Bergue C.T., Fauth G. 2009. Maastrichtian-Early Danian radiolarians and Ostracodes from ODP Site 1001B, Caribbean Sea. Revista Brasileira de Paleontologia, 12(3):195210.

Bogdanov N.A., Vishnevskaya V.S. 1992. Influence of evolutionary changes in Radiolaria on sedimentary processes. Doklady Akademii Nauk SSSR, 324:162166.

Boltovskoy D. 1998. Classification and distribution of South Atlantic Recent polycystine Radiolaria, Paleontologia Electronica, 1(2):URL: < http://palaeoelectronica.org/1998_2/boltovskoy/issue2.htm> Acesso: 01.07.2010.

Boltovskoy D., Pujana I. 2007. Radiolaria. In: Camacho H. ed. 2007. Invertebrados Fósiles. Buenos Aires: Univ. Buenos Aires Maimónides, p. 111132.

Boltovskoy D., Riedel W.R. 1987. Polycystine Radiolaria of the California current region: seasonal and geographic patterns. Marine Micropaleontology, 12(1):65-104

Boltovskoy D., Alder V., Abelmann A. 1993. Annual flux of Radiolaria and other shelled plankters in the eastern equatorial Atlantic at $853 \mathrm{~m}$ : seasonal variations and Polycystine species-specific response. Deep-Sea Research, 40(9):1863-1895.

Boltovskoy D., Kogan M., Alder V., Mianzan, M. 2003. First record of a brackish radiolarian (Polycystina): Lophophaena rioplatensis $\mathrm{n}$. sp. in the Río de la Plata estuary.Journal of Plankton Research, 25:1551-1559.

Cachon J., Cachon M. 1971. Recherches sur les metabolism de la silice chez les radiolaires. Absorption et excrétion. Comptes rendus de l'Académie des Sciences (Paris), Série II:272:16521654.

Campbell A.S. 1954. Radiolaria. In: Moore R.C. ed. 1954. Treatise on Invertebrate Paleontology. Part D, Protista 3: Protozoa (chiefly Radiolaria and Tintinnina). Lawrence: Geological Society of America and University of Kansas Press, p. 11163.

Carter E.S., Haggart J.W. 2006. Radiolarian biogeography of the Pacific region indicates a mid- to high-latitude $\left(>30^{\circ}\right)$ position for the Insular superterrane since the late Early Jurassic. In: Haggart J.W., Enkin R.J., Monger J.W.H. eds. 2006. Paleogeography of the North American Cordillera: Evidence For and Against Large-Scale Displacements.
Ottawa: Geological Association of Canada. p. 109132. (Special Paper 46).

Casey R.E., Wigley C.R., Perez-Guzmann A.M. 1983. Biogeographic and ecologic perspective on polycystine radiolarian evolution. Paleobiology, 9:363-376.

Cortese G., Abelmann A. 2002. Radiolarian-based paleotemperatures during the last $160 \mathrm{kyr}$ at ODP Site 1089 (Southern Ocean, Atlantic Sector). Palaeogeography, Palaeoclimatology, Palaeoecology, 182:259-286.

De Wever P., Dumitrica P., Caulet J.P., Nigrini C., Caridroit M. 2001. Radiolarians in the sedimentary record. Amsterdam: Gordon \& Breach Science Publ. 533p.

Dumitrica P., Hollis C.J. 2004. Maastrichtian Challengeriidae (Phaeodarian Radiolaria) from deep sea sediments of SW Pacific. Revue de Micropaléontologie, 47:127-134.

Ehrenberg C.G. 1875. Fortsetzung der mikrogeologischen Studien als GesammtÜbersicht der mikroskopischen Paläontologie gleichartig analysirter Gebirgsarten der Erde, mit specieller Rucksicht auf den PolycystinenMergel von Barbados. Königliche Akademie der Wissenschaften zu Berlin, Abhandlungen, 1-225.

Eilert V.P. 1985. Ocorrência da Polycystina (Protozoa, Radiolaria) antárticos e subantárticos em sedimentos superficiais do Atlântico Sudoeste $\left(31^{\circ}-35^{\circ} \mathrm{S} ; 45^{\circ}-54^{\circ} \mathrm{W}\right)$. Pesquisas, 17:151-167.

Eilert V.P. 2001. Polycystine radiolarians in Brazilian sedimentary basins: a synthesis of the current status. Revista Brasileira de Paleontologia, 2:7-19.

Eilert V.P., Baecker-Fauth S., Souza V de. 2004. Radiolários. In: Carvalho, I. de S. ed. 2004. Paleontologia. Rio de Janeiro: Editora Interciência. p. 297-312.

Funakawa S., Nishi H. 2005. Late middle Eocene to late Oligocene radiolarian biostratigraphy in the Southern Ocean (Maud Rise, ODP Leg 113, Site 689). Marine Micropaleontology, 54(3-4):213-247.

Funnell B.M., Riedel W.R. 1971. The Micropalaeontology of Oceans. Cambridge: Cambridge Univ. Press. $828 \mathrm{p}$.

Haeckel E. 1887. Report on the Radiolaria collected by H.M.S. Challenger during the years 1873-1876. Johnson Reprint Corporation. clxxxviii+1803p. (Report on the scientific results of the voyage of the H.M.S. Challenger, Zoology, 18).

Hallam A., Wignall P. 1997. Mass Extinctions and their Aftermath. Oxford: Oxford Univ. Press. 320 p.

Imbrie J., Kipp. N.G. 1971. Anew micropaleontological method for quantitative paleoclimatology: Application to a late Pleistocene Caribbean core. In: Turekian K. K. ed. 1971. The Late Cenozoic Glacial Ages. New Haven: Yale Univ. Press. p. 71-181. 
Jacquot Des Combes H., Abelmann A. 2009. From species abundance to opal input: Simple geometrical models of radiolarian skeletons from the Atlantic sector of the Southern Ocean. DeepSea Research - Part I, 56:757-771.

Jacquot Des Combes H., Caulet J.P., Tribovillard N. 1999. Pelagic productivity changes in the equatorial area of the NW Indian Ocean during the last $350 \mathrm{ka}$. Marine Micropaleontology, 158:2755.

Kattah S.S., Koutsoukos E.A.M. 1992. A ocorrência de radiolários em fácies sedimentares de origem marinha no Mesozóico da Bacia Sanfranciscana. Rev. Esc. Minas, 45(1/2):214.

Kochhann K.G.D., Baecker-Fauth S., Fauth G., Silveira A.S. 2009. Aalenian (Early Jurassic) radiolarian fauna from Los Molles Formation, Argentina, and its paleobiogeographic affinities. In: XXI Congresso Brasileiro de Paleontologia, 2009, Belém - Pará. Livro de Resumos, 1:p. 39.

Kotzian S.C.B. 1984. Radiolários (Polycystina) pleisto-holocênicos do testemunho T15. Margem Continental Sul-brasileira (333' $33^{\prime}$ $\left.48^{\circ} 28^{\prime} \mathrm{W}\right)$. In: Congresso Brasileiro de Geologia, 1984, Rio de Janeiro - Rio de Janeiro. Anais, 3:627-644.

Kotzian S.C.B., Eilert V.P. 1985. Radiolários Polycystina (Spumellaria: Sponguridae, Spongodiscidae) cenozóicos em testemunhos da Margem Continental Sul-brasileira. Coletânea de Trabalhos Paleontológicos (Brasília: DNPM, Ministério das Minas e Energia), 27:34-53.

Kotzian S.C.B., Eilert V.P., Ruthner M. 1987. Nova contribuição sobre a ocorrência de radiolários Polycystina na margem continental do Rio Grande do Sul, Brasil. Geomar VI. Pesquisas, 20:85-102.

Mizutani S. 1966. Transformation of silica under hydrothermal conditions. Nagoya Journal of Earth Sciences, 14:56-88.

Müller J. 1858. Über die Thalassicollen, Polycystinen und Acanthometren des Mittelmeeres. Königliche Preussische Akademie der Wissenschaft zu Berlin, Abhandung, 1-62.

Nigrini C. 1967. Radiolaria in pelagic sediments from the Indian and Atlantic oceans. Bulletin of the Scripps Institute of Oceanography, 11:1-125.

Ogane K., Tuji A., Suzuki N., Kurihara T., Matsuoka A. 2009. First application of PDMPO to examine silicification in polycystine Radiolaria. Plankton E Benthos Research, 4(3):89-94.
Pessagno E.A. Jr., Blome C.D. 1986. Faunal affinities and tectonogenesis of Mesozoic rocks in the Blue Mountains province of eastern Oregon and western Idaho. In: Vallier T.C., Brooks H. eds. 1986. Geology of the Blue Mountains Region of Oregon, Idaho, and Washington. Geologic Implications of Paleozoic and Mesozoic Paleontology and Biostratigraphy, Blue Mountains Province. US Geological Survey, Professional Paper 1435, pp. 65-78.

Pessagno E.A. Jr., Hull D.M., 2002. Upper Jurassic (Oxfordian) Radiolaria from the Sula Islands (East Indies): Their taxonomic, biostratigraphic, chronostratigraphic and paleobiogeographic significance. Micropaleontology, 48:229-256.

Racki G. 1999. Silica-secreting biota and mass extinctions: survival patterns and processes. Palaeogeography, Palaeoclimatology, Palaeoecology, 154:107-132.

Sandoval J., O‘dogherty L., Aguado R., Bartolini A., Bruchez S., Bill M. 2008. Aalenian carbonisotope stratigraphy: Calibration with ammonite, radiolarian and nannofossil events in the Western Tethys. Palaeogeography, Palaeoclimatology, Palaeoecology, 267:115-137.

Swain F.M. 1977. Stratigraphic Micropaleontology of Atlantic Basin and Borderlands. New York: Elsevier. 603 p.

Takemura A. 1992. Radiolarian Paleogene biostratigraphy in the Southern Indian Ocean, Leg 120. In: Schlich R., Wise S.W., Julson A.A.P., Aubry M.P., Berggren W.A., Bitschene P.R., Blackburn N.A., Breza J., Coffin M.F., Harwood D.M., Heider F., Holmes M.A., Howard W.R., Inokuchi H., Kelts K., Lazarus D.B., Mackensen A., Maruyama T., Munschy M., Pratson E., Quilty P.Q., Rack F., Salters V.J.M., Sevigny J.H., Storey M., Takemura A., Watkins D.K., Whitechurch H., Zachos J. eds. 1992. Proceedings of the Ocean Drilling Program, Scientific Results, 120. College Station: Texas A\&M University. p. 735-756.

Thompson W.D.A. 1942. On growth and form. Cambridge: Cambridge University Press. 1116 p.

Won M.Z., Below R. 1999. Cambrian Radiolaria from the Georgina Basin, Queensland, Australia. Micropaleontology, 45:325-63. 